\title{
Numerical study of the radio microstructure associated with the plasma turbulence emission model
}

\author{
James C. Weatherall \\ New Mexico Institute of Mining and Technology
}

\begin{abstract}
Recent observational data of short time-scale fluctuations in the radio emission of pulsars offer the opportunity for new tests of proposed emission models. For this purpose, the existing models must be developed to make predictions regarding the temporal characteristics of the emission. A computer solution of the time evolution of plasma wave turbulence details the properties of the nonlinear plasma emission mechanism. As a consequence of ponderomotive nonlinearity in the plasma medium, two-stream growing modes develop modulational instability, leading to the onset of strong, two-dimensional plasma turbulence. The turbulence exhibits explosive spatial collapse of regions of high electric field, and the escape of bursts of radiation.
\end{abstract}

\section{Introduction}

Radio microstructure of pulsars is the extremely fine temporal fluctuation observed at less than 1 microsecond. At $100 \mathrm{~ns}$ or $10 \mathrm{~ns}$ time scales, microstructure may sample the dynamical properties of the coherence process underlying the emission. Because observers are now able to resolve these time structures (Hankins - these proceedings), we have the opportunity to apply new tests of theoretical models against observational data.

Another reason to focus on microstructure is that it divorces the emission physics from the magnetospheric and global physics. One can study the well defined (maybe even tractable) problem of how a strongly magnetized pair plasma. radiates without taking on the complicated problem of how the "machine" works.

The new theoretical challenge is to derive the temporal characteristics of the radiation signal implied by existing emission models. The following description of the microstructure predicted by the nonlinear plasma emission mechanism (e.g. Asseo et al. 1990) is based on numerical solution of coupled wave equations for plasma turbulence.

\section{Plasma Turbulence Model}

The evolution of electric field and plasma density is solved on a two-dimensional $64 \times 64$ point $x y$ spatial grid by computer. The fields are Fourier-decomposed into $42 \times 42$ (aliased) wavemodes. The dispersion and polarization properties of these modes are modeled by the linear characteristics of the ordinary mode (fast branch) solution of the Maxwell matrix equation in the limit of infinite magnetic 
field, $B_{y}$, in a $e^{+} e^{-}$plasma. The dispersion relation of this mode is given by (Arons \& Barnard 1986):

$$
\omega^{2}(\vec{k})=\frac{1}{2} \omega_{p}^{2}\left[1+\frac{c^{2} k^{2}}{\omega_{p}^{2}}+\left[\left(1+\frac{c^{2} k^{2}}{\omega_{p}^{2}}\right)^{2}-4 \frac{c^{2} k_{y}^{2}}{\omega_{p}^{2}}\right]^{1 / 2}\right]
$$

This mode's electric field polarization is predominantly along the magnetic field direction, $\hat{e}_{y}$, when $k_{y}<\omega_{p} / c$, and couples strongly to the plasma which is constrained by the magnetic field to move along the $y$-direction. The wavemode is a plasma oscillation for wavevectors in the direction of the magnetic field, and a transverse vacuum electromagnetic wave for wavevectors perpendicular to the magnetic field. For other angles, the wavemode is neither pure transverse nor longitudinal.

Numerical solution is made of the coupled modal equations for Fourier-space amplitudes $E_{k_{x}, k_{y}}$ :

$$
\left(\frac{d^{2}}{d t^{2}}+w_{k}^{2}\right) E_{k_{x}, k_{y}}=\omega_{p}^{2}\left(\Lambda E_{y}\right)_{k_{x}, k_{y}} c_{k_{x}, k_{y}}
$$

where $c_{k_{x}, k_{y}}$ is a geometric coupling term. The nonlinear coupling is due to variation in the index of refraction, where $\Lambda=-\delta n / n$. When the modal amplitude varies on a slower time scale than the phase oscillation near the plasma frequency, the density change is determined by the ponderomotive potential, given by $\Lambda=\left\langle E_{y}^{2}-\vec{E}_{y}^{2}\right\rangle /\left(32 \pi N m v_{t h}^{2}\right)$, where $<>$ denotes time average, and the overbar is a spatial average. Further exploitation of the separable time scales can recast the modal equation as a nonlinear Schrödinger equation, which has soliton solutions. A split-step Fourier algorithm is applied for numerical solution to the coupled mode equations (Hardin \& Tappert 1973).

\section{Evolution of plasma turbulence in the pulsar magnetosphere}

Computer solution for the development of turbulence in the pulsar source region is exhibited in Figure 1, which shows surface plots of the in-situ $E_{y}$ field on the $x y$ spatial grid at various times. The spatial extent of the grid is $64 \times 0.6 c /\left(2 \pi f_{p}\right)$.

The turbulence originates with the plasma two-stream instability (Cheng \& Ruderman 1970). To simulate a beam-plasma instability in the computer code, a single wavemode with $k_{y}=\omega_{p} / c$ is incremented each time step at a rate $\gamma=0.001 \omega_{p}$. This unstable wavemode is a plasma wave propagating in the magnetic field direction with a phase velocity resonant with relativistically streaming particles. Figure 1a shows this mode in the pre-turbulent system.

At large amplitudes, the beam-resonant mode is modulationally unstable, as can be shown from an analytic linear instability analysis. The instability is seen as a transverse ripple on the beam mode in Figure $1 \mathrm{~b}$. The modulational instability enters a nonlinear stage, in which the ripple steepens and becomes nonsinusoidal. This evolves to a strongly turbulent state in which wavepackets 'collapse' to smaller spatial dimension, producing a turbulent cascade which 

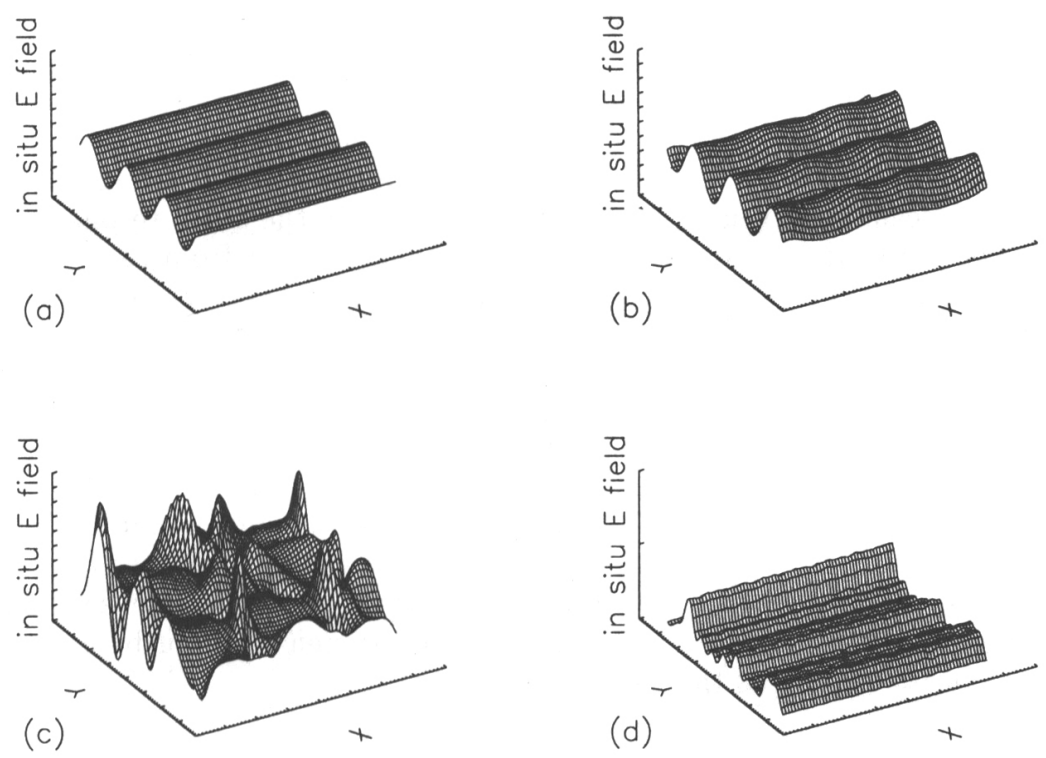

Figure 1. Evolution of electromagnetic turbulence depicted by $y-$ component of electric field in two spatial dimensions; a) during growth of beam unstable mode; b) onset modulational instability of beam mode; c) fully developed two-dimensional turbulence; d) onedimensional turbulence showing solitary wave structures.

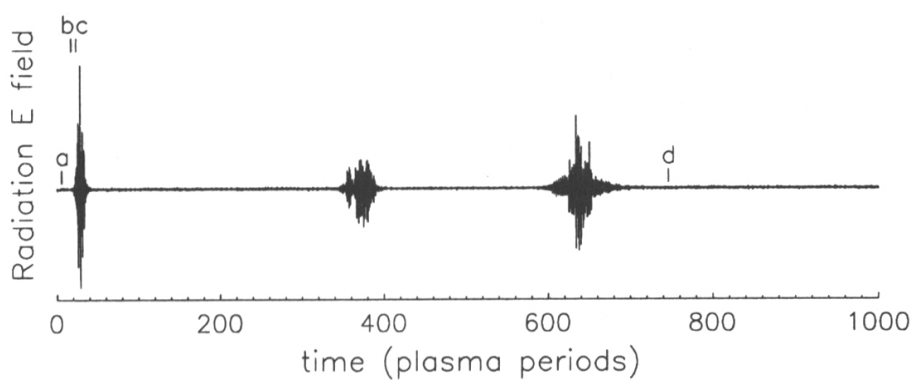

Figure 2. Time history of radiation escaping simulation volume; times corresponding to Figure 1 are marked. 
involves wavemodes of various wavelength scales. Figure 1c shows the fully developed turbulent state.

During the turbulent stage, energy is transported to radiative modes, i.e., modes which have finite Poynting flux. By removing energy from the boundary of the source region, these modes are dissipative. This damping is modeled in the computer code by damping at a rate $\gamma=-0.1 \hat{E} \times \hat{B} \omega_{p}$; this corresponds to the rate of radiative loss from a source region of radius $40 c /\left(2 \pi f_{p}\right)$. The turbulent cascade is driven at the expense of energy in the beam resonant mode. As the beam resonant mode is depleted, and energy is lost from the system via radiation, the turbulent cascade is shut off. The wavemode sum of the escaping E-field seen along the $\mathrm{x}$-axis is shown in Figure 2. The radiation is intense during time of two-dimensional turbulence.

After quenching of the two-dimensional turbulence, the fields revert to a one-dimensional state in which soliton wave structures (Asseo et al. 1990) appear. An example of one-dimensional turbulence is shown in Figure 1d. Radiation by these structures is discussed by Asseo et al., but is not included in the physics of the computer model. As the beam instability pumps energy back into the system, transitions to two-dimensional turbulence and intense radiation occur intermittently, separated by periods of one-dimensional turbulence. This process repeats as long as the code is run.

\section{Conclusion}

Plasma turbulence is seen as a turbulent cascade by which energy is injected into electrostatic modes and transported into radiative modes. The escaping radiation shows structure on several timescales: the interval between pulses is $200 / f_{p}$, as established by the beam instability growth. The width of the pulse, $50 / f_{p}$, is the dynamical timescale of the nonlinear turbulent cascade. Finally, modulational structure is seen over $10 / f_{p}$. For $f_{p}=5 \mathrm{GHz}$, these timescales are 40,10 , and $2 n s$, respectively. These numbers are model dependent, so a range of parameter space relating to instability growth rates and source volume needs to be explored. The theoretical calculation provides new data to compare with observations of radio microstructure.

Acknowledgments. My thanks to Jean Eilek and Tim Hankins for useful discussions, and for support from NSF grant AST-9315285 and NMIMT.

\section{References}

Arons, J. \& Barnard, J.J. 1986, ApJ, 302, 120

Asseo, E., Pelletier, G., \& H. Sol 1990, MNRAS, 247, 529

Cheng, A.F. \& Ruderman, M.A. 1977, ApJ, 214, 598

Hardin, R., \& Tappert, F. 1973, SIAM Rev, 15, 423 